\title{
Average Error Probability in Wireless Sensor Networks With Imperfect Sensing and Communication for Different Decision Rules
}

\author{
Pedro Henrique Juliano Nardelli, Iran Ramezanipour, Hirley Alves, \\ Carlos H. M. de Lima, and Matti Latva-Aho
}

\begin{abstract}
This paper presents a framework to evaluate the probability that a decision error event occurs in wireless sensor networks, including sensing and communication errors. We consider a scenario where sensors need to identify whether a given event has occurred based on its periodic, noisy, and observations of a given signal. Such information about the signal needs to be sent to a fusion center that decides about the actual state at that specific observation time. The communication links-singleor multi-hop-are modeled as binary symmetric channels, which may have different error probabilities. The decision at the fusion center is based on OR, AND, $K$-OUT-OF- $N$, and MAJORITY Boolean operations on the received signals associated to individual sensor observations. We derive closed-form equations for the average decision error probability as a function of the system parameters (e.g., number of sensors and hops) and the input signal characterization. Our analyses show the best decision rule is closely related to the frequency that the observed events occur and the number of sensors. In our numerical example, we show that the AND rule outperforms MAJORITY if such an event is rare and there is only a handful number of sensors. Conversely, if there are a large number of sensors or more evenly distributed event occurrences, the MAJORITY is the best choice. We further show that, while the error probability using the MAJORITY rule asymptotically goes to 0 with increasing number of sensors, it is also more susceptible to higher channel error probabilities.
\end{abstract}

Index Terms-Data fusion, distributed detection, wireless sensor networks.

\section{INTRODUCTION}

$\mathbf{I}$ N A RECENT report, the consulting group McKinsey claims that the Internet of Things (IoT) - "sensors and actuators connected by networks to computing systems" have "a total potential economic impact of $\$ 3.9$ trillion to

Manuscript received January 15, 2016; revised February 16, 2016; accepted February 25, 2016. Date of publication February 29, 2016; date of current version April 8, 2016. This work was supported in part by the Suomen Akatemia within the Strategic Research Council through the Aka BC-DC Project under Grant 292854, in part by the Luonnontieteiden ja Tekniikan Tutkimuksen Toimikunta under Grant 271150, and in part by the Conselho Nacional de Desenvolvimento Científico e Tecnológico and Brazil, through the Joint Project SUSTAIN under Grant 490235/2012-3. The associate editor coordinating the review of this paper and approving it for publication was Prof. I. Koo.

P. H. J. Nardelli, I. Ramezanipour, H. Alves, and M. Latva-Aho are with the Centre for Wireless Communications, University of Oulu, Oulu 90014, Finland (e-mail: nardelli@ee.oulu.fi; ramezanipour@gmail.com; halves@ee.oulu.fi; matla@ee.oulu.fi).

C. H. M. de Lima is with São Paulo State University, São Paulo 15054-000, Brazil (e-mail: carlos.lima@sjbv.unesp.br).

Digital Object Identifier 10.1109/JSEN.2016.2536148
$\$ 11.1$ trillion a year by 2025" [1]. Although these numbers and methodology might be questionable, the fact that wireless sensor networks (WSNs), which build the core of IoT, are becoming widespread in almost any possible application area, ranging from energy systems to sleep monitoring [2].

This wide variety of uses implies the nonexistence of a given optimal technology solution that fits all WSNs (e.g. [2], [3]); the requirements of control applications in industries (e.g. [4]) is far different from in household monitoring (e.g. [5]) and the WSN deployment should reflect these differences.

In this paper, we will focus on general applications with loose reliability requirements so that sensors may have limited computational capabilities. Our goal here is to understand how to build a more efficient WSN to detect a given event based on an input signal, keeping its design as simple as possible. We follow here the idea of distributed detection (e.g. [6], [7]) so that every sensor estimates the occurrence of such an event and then sends this information to a fusion center, which decides based on the locally processed data; in contrast, by employing a centralized approach, the sensors need to send all their raw observations to the fusion center that then makes a decision.

In signal processing and information theory [8], the field of distributed detection and estimation, which has a relatively long history (e.g. [8]-[13]), builds an elegant theoretical framework for finding optimal (locally and globally) strategies. In a series of works dating back to the 80's and 90's, the core theoretical findings of distributed detection were established, as summarized in [11]. Therein, the authors reviewed the advances on the topic, pointing out three different network topologies: parallel (sensors are not connected to one another), serial (sensors connected in series) and tree (sensors connected following a tree hierarchy). Different formulations for the detection problem have been then described and their optimal solutions discussed.

For instance, the Neyman-Pearson formulation poses the problem as follows [11]: "for a prescribed bound on the global probability of false alarm, find (optimum) local and global decision rules that minimize the global probability of miss." Other way of formulating the problem is based on Bayesian statistics aiming at minimizing the Bayes risk. Although they differ in basic aspects, both search ways of optimizing the detection rules based on binary hypothesis tests on the 
presence of a given signal and they state the likelihood-ratio test as the optimal rule (with different parameters, though). For these cases, the analyses are usually carried out in terms of false positives (also known as type I error, or false-alarm) and false negatives (also known as type II errors, or misdetection).

Even though these results have been established for decades, there is still a great interest in distributed detection. Recently, Zhang et al. studied in two subsequent papers [14], [15] the detection error probability in balanced binary relay trees. The leaves of the trees (lowest level) are related to the sensors while the root (highest level) is the fusion center that makes the decision. In between them, relay nodes combine the binary messages sent by their two neighbors at the lower level (either sensors if we consider the second lowest level or other relays for the other levels). Using the likelihood test at every level, they derive tight bounds of the error probability as a function of the number of sensors considering the binary symmetric channel [14] and erasure channel [15]. In [16], the authors assess the probability of decision error of a network with noisy binary symmetric channels between sensors and fusion center and points out that the fusion rule should be optimized with respect to the observed phenomenon.

In [17], the authors combine the idea of robust decentralized detection with social learning. Among its contributions, [17] generalizes to tree and tandem topologies the results of [18], where a solution to the "minimax" robust detection problem (whose objective is to minimize the worst case performance when the probability distribution of the observations is not completely specified) for parallel topology is presented. In a recent paper [19], the authors combine the advances in spatial models for wireless networks using spatial point process theory (e.g. [20] and references therein) and distributed detection. In [21], decentralized detection for clustered sensor networks with hierarchical multi-level fusion is investigated. Authors conclude that the probability of decision error is dominated by the number of decision levels rather than the clustering formation, which renders minimum performance degradation with uniform clustering. In addition to wireless sensor networks, distributed detection has been applied in the analysis of spectrum sensing strategies for cognitive radio networks as in [22].

The field of distributed estimation, although similar, has received relatively less attention. We can cite the following papers as initial works on that problem [9], [10], [23]. More recently in [24] and [25], the authors studied the case of distributed estimation with constrained bandwidth of 1 bit, proposing a class of maximum likelihood estimators. Other relatively new results can be found in [26] and [27], while [13] provides an interesting survey on the topic.

In this paper, we choose a slightly different way by analyzing a scenario where the quantization and decision rules are given, instead of seeking for optimal detection/estimation schemes [7]. As previously mentioned, our aim is to assess the average error probability of a WSN with little computational capabilities. Specifically, our scenario is a set of sensors that periodically measure a given signal to detect whether a given event happens (e.g. if the signal has a value above a certain threshold). Based on their noisy measurements, the occurrence of such events is mapped into a binary number (e.g. occurrence implies "1", not occurrence implies " 0 "), which defines the sensor state. The sensors need then to send their states to the fusion center via wireless channel, through one or multiple hops. The relay nodes only forward the information they received. We assume binary symmetric channels whose associated error probabilities might be different at each level of the multi-hop transmission (but, within the same level, the probabilities are the same). A decision about the state of the signal is done at the fusion center based on the binary signals related to each sensor. The decision rules employed by the fusion center are the memoryless Boolean functions OR, AND, $K$-OUT-OF- $N$ and MAJORITY.

Our study targets at answering the following: Under which conditions a low average error probability can be achieved for the scenario described above? For example, the answer for this question for the case of rare events is that a combination of AND decision rule by the fusion center and quantization of the event occurrence with " 1 " leads to an average error probability close to the event frequency itself, even when only a handful of sensors are used. A deeper discussion about this is found later in this paper. It also important to mention that our work differs from others in the literature as [16] and [21] by focusing on a single error probability metric rather than cross-over, falsepositive or false-negative probabilities. More specificaly, the present work generalizes [16] to multi-hop binary symmetric channels and general fusion rules.

In summary, we identify the following main contributions: (i) propose an analytic framework that first breaks the WSN into three phases - sensing, communication and decision to analyze the error events related to each one of them (Section II) to then rebuild the system as a whole to understand how the error propagates through those phases (Section III), regardless of the input function; (ii) find a joint error probability which accounts for sensing and communication, while considering that the sensor observations (which are conditionally independent) are subject to Gaussian noise and independent binary symmetric channels with different error probabilities at each hop (Section II); ( $i$ ii) derive, in closed-form, the average error probability for the OR, AND, $K$-OUT-OF- $N$ and MAJORITY (Section III), showing that MAJORITY rule can asymptotically reach a 0 -error probability with the number of sensors; (iv) show that the performance of OR and AND rules depends on the frequency of the event under analysis, while the other two do not - this fact implies that, when a limited number of sensors is considered, OR or AND rule (depending on the quantization mapping) can outperform the MAJORITY; (v) exemplifying the analysis with numerical results (Section IV) to illustrate our findings, providing the basis for our final discussions and possible extensions (Section V).

\section{System DESCRIPTION}

Let us assume a network composed by a set $\mathcal{N}=$ $\{1, \ldots, N\}$ of sensors that monitor a continuous signal $x(t)$, where $t \in \mathbb{R}^{+}$and $x: \mathbb{R}^{+} \rightarrow \mathbb{R}$, to estimate whether a given event $\mathcal{E}$ related to $x(t)$ happens and send this information to a fusion center. To avoid confusion, we summarize the key notations in Table I. Assuming that the sensors make 
TABLE I

NOTATION SUMMARY

\begin{tabular}{|c|l|}
\hline Notation & Description \\
\hline \hline $\mathcal{N}$ & set of sensors \\
\hline$N$ & number of sensors in set $\mathcal{N}$ \\
\hline$M$ & number of communication hops \\
\hline$\eta$ & total number of samples \\
\hline$x(t)$ & continuous signal as a function of time $t$ \\
\hline$x_{\text {th }}$ & signal threshold \\
\hline$\theta[n]$ & sensor state at time $t_{n}$ related to the $n$th measurement \\
\hline$y_{i}[n]$ & sensor $i$ estimation about the $n$th measurement \\
\hline $\mathcal{E}$ & event related to $x(t)$ \\
\hline$s_{i}[n]$ & received state at the fusion center from sensor $i$ \\
\hline$g(\cdot)$ & decision function \\
\hline$\hat{\theta}[n]$ & estimated stated at the fusion center \\
\hline$n_{i}\left(t_{n}\right)$ & additive noise associated with sensor $i$ at $t_{n}$ \\
\hline$\mu$ & noise mean \\
\hline$\sigma^{2}$ & noise variance \\
\hline$S$ & Boolean variable associated with event $\mathcal{E}$ \\
\hline$f_{S}$ & frequency of a given state $S s$ \\
\hline$S_{i, j}$ & state of sensor $i$ in hop $j$ \\
\hline$p_{j}$ & error probability in the communication hop $j$ \\
\hline $\mathcal{C}_{M}$ & set of all possible error events for $M$-hop link \\
\hline
\end{tabular}

synchronous and periodic measurements in predetermined instants $t_{n}=n \tau$ with $n$ being a natural number and $\tau \in \mathbb{R}^{+}$, we can define a function $\theta[n]$ with $\theta: \mathbb{N} \rightarrow\{0,1\}$ that indicates if $\mathcal{E}$ occurs at time $t_{n}$. Hereafter we refer to $\theta[n]$ as the system state at time $t_{n}$.

The sensors' estimation of $\theta[n]$ is, however, imperfect. For each sensor $i \in \mathcal{N}$, we define a function $y_{i}[n]$ with $y_{i}: \mathbb{N} \rightarrow\{0,1\}$ that represents the estimation about $\mathcal{E}$ from its individual noisy version of $x(t)$. If a sensing error at sensor $i$ happens at $t_{n}$, then $y_{i}[n] \neq \theta[n]$; otherwise $y_{i}[n]=\theta[n]$.

After the sensing phase, the sensors need then to forward $y_{i}[n]$ to a remotely located fusion center, which will process the received information to determine whether $\mathcal{E}$ has indeed occurred. Each sensor $i \in \mathcal{N}$ sends its state $y_{i}[n]$ through independent communication channels that are also subject to errors. Let $s_{i}[n]$ with $s_{i}: \mathbb{N} \rightarrow\{0,1\}$ be the state related to sensor $i$ that is received by the fusion center after passing through the radio links, which can be composed by only one hop or multiple hops where relay nodes forward the received information. If an error occurs in the link related to sensor $i$ 's $n$th measurement, then $s_{i}[n] \neq y_{i}[n]$; if not, $s_{i}[n]=y_{i}[n]$.

From the signals $s_{i}[n]$, the fusion center needs to decide whether event $\mathcal{E}$ happened at $t_{n}$. Let $g\left(s_{1}[n], \ldots, s_{N}[n]\right)$, with $g:\{0,1\}^{N} \rightarrow\{0,1\}$, denote the Boolean function that estimates the state $\theta[n]$ by the fusion center so that the estimated state $\hat{\theta}[n]$ related to $t_{n}$ is given by $\hat{\theta}[n]=g\left(s_{1}[n], \ldots, s_{N}[n]\right)$. A decision error occurs whenever $\hat{\theta}[n] \neq \theta[n]$. The average error probability $P_{\mathrm{e}}$ of the whole process is given by:

$$
P_{\mathrm{e}}=\frac{1}{\eta} \sum_{n=0}^{\eta-1} \operatorname{Pr}[\hat{\theta}[n] \neq \theta[n]],
$$

where the average is taken over the samples $n \in\{0,1, \ldots$, $\eta-1\}$ related to a time window from $t_{0}=0$ and $t_{\eta-1}=T$.

Our goal here is to analyze different design options for the sensor network and the fusion center's decision function to

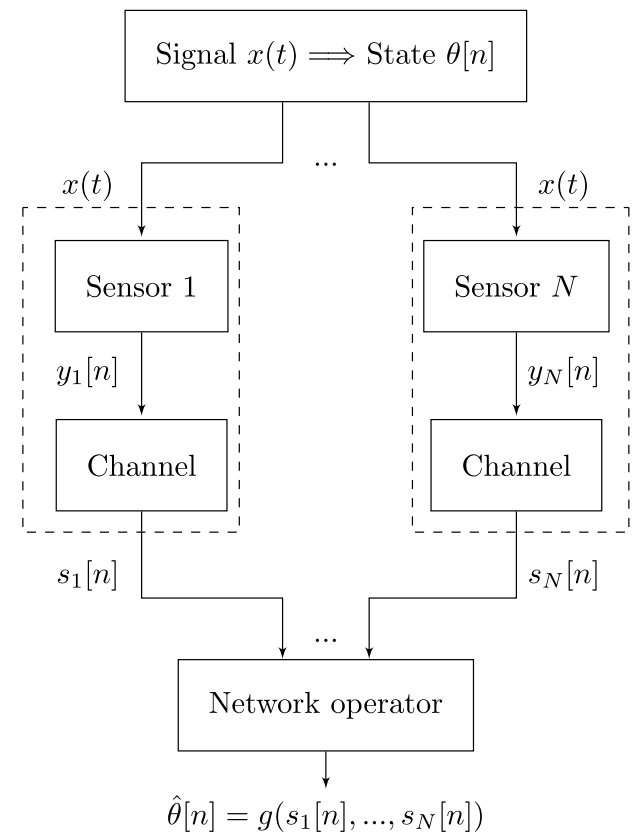

Fig. 1. Illustrative figure of the scenario under analysis. Sensors monitor a given signal $x(t)$ in order to determine the binary state $\theta[n]$ at time $t_{n}$. Each one of the $N$ sensors in the network needs to send its state to a fusion center (control unit) that remotely decides the state $\hat{\theta}[n]$. In its way to the fusion center, errors may happen either in sensing $\left(y_{i}[n] \neq \theta[n]\right.$ with $\left.i=1, \ldots, N\right)$ or in communicating $\left(s_{i}[n] \neq y_{i}[n]\right)$. The dashed rectangle identifies where the error events may happen. Our goal is to find the expected error probability $P_{\mathrm{e}}$ given by (1) and then compare different design options.

improve the estimation reliability, evaluated by the probability that $\hat{\theta}[n] \neq \theta[n]$. Fig. 1 illustrates the scenario under analysis. For instance, $x(t)$ may represent the temperature of an industrial plant that requires temperatures below a given threshold $x_{\text {th }}$ to guarantee its safe operation. The event $\mathcal{E}$ can be then associated with an emergency where $x\left(t_{n}\right)>x_{\text {th }}$. Given the signal $x(t)$, the threshold $x_{\text {th }}$ and the number of sensors $N$, we need to find the most suitable design for the quantization function $\theta$ (i.e. define if the event $x(t)>x_{\text {th }}$ is associated with $\theta=0$ or $\theta=1$ ) and the decision rule $g$ (OR, AND, $K$-OUT-OF- $N$, MAJORITY) at the fusion center.

Next we will focus our attention on the errors in the sensing procedure and in the communication links, which are identified by the dashed rectangle in Fig. 1, and how they affect the decisions done by the fusion center.

\section{A. Sensing Error}

Let us denote $x_{i}\left(t_{n}\right)$ the version of $x(t)$ observed by sensor $i \in \mathcal{N}$. The value of $x_{i}\left(t_{n}\right)$ will be then used to define $y_{i}[n]$. Then, we can define the probability $P\left(x_{i}\left(t_{n}\right) \leqslant x_{\text {th }}\right)$ that the event $x_{i}\left(t_{n}\right) \leqslant x_{\mathrm{th}}$, and its complement $P\left(x_{i}\left(t_{n}\right)>x_{\mathrm{th}}\right)$.

Remark 1: The sensing error probability is dependent on the input signal $x(t)$ such that an error in the sensing procedure $y_{i}[n] \neq \theta[n]$ occurs in two situations: (a) $x\left(t_{n}\right)>x_{\text {th }}$ and $x_{i}\left(t_{n}\right) \leqslant x_{\text {th }}$, or (b) $x\left(t_{n}\right) \leqslant x_{\text {th }}$ and $x_{i}\left(t_{n}\right)>x_{\text {th }}$. The error probability is then related to the frequency that $x\left(t_{n}\right)$ is above or below the threshold $x_{\text {th }}$, which is captured by how many times $\theta[n]=0$ or $\theta[n]=1$ for $n=0, \ldots, \eta-1$. 


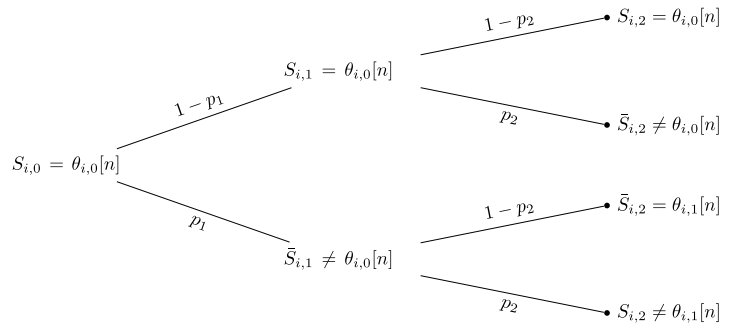

Fig. 2. State probability tree for each sensor $i=1, \ldots, N$ considering communication error probabilities $p_{j}$ with $j=1, \ldots, M$ where $M$ is the number of hops between the sensors and the fusion center. The initial state is $S_{i, 0}=y_{i}[n]$ and the final state is $S_{i, M}$. The state $S_{i, j} \in\{0,1\}$ and $\bar{S}_{i, j}$ denotes its complement.

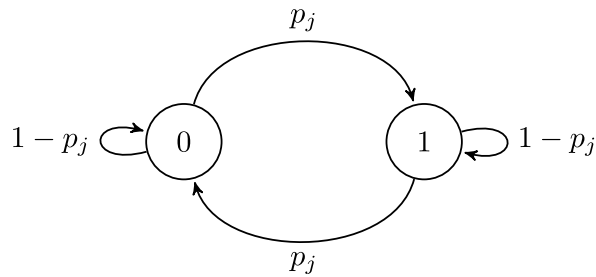

Fig. 3. Transition diagram between the two possible states " 0 " and " 1 " when the Binary Symmetric Channel is assumed. A change only occurs with probability $p_{j}$ with $j=1, \ldots, M$, which is associated with the $j$ th level error probability such that $S_{i, j}=S_{i, j-1}$ with probability $1-p_{j}$ and $S_{i, j} \neq S_{i, j-1}$ with probability $p_{j}$.

Let us consider $x\left(t_{n}\right) \leqslant x_{\text {th }}$ is associated to the state $\theta[n]=S$ where $S \in\{0,1\}$ and $x\left(t_{n}\right)>x_{\text {th }}$ is associated to the state $\theta[n]=\bar{S}$, where $\bar{S}$ denotes the complement of $S$. Then, we have the following definition.

Definition 1: Recalling that $\eta$ denotes the total number of samples within an arbitrary interval defined by $t_{0}=0$ and $t_{\eta-1}=T$. We define $\eta_{S} \triangleq \eta_{x\left(t_{n}\right) \leqslant x_{\mathrm{th}}}$ and $\eta_{\bar{S}} \triangleq \eta_{x\left(t_{n}\right)>x_{\mathrm{th}}}$ as the number of samples related to states $S$ and $\bar{S}$ in such interval, respectively. In this case, $\eta_{S}+\eta_{\bar{S}}=\eta$. The frequency $f_{S}=f_{x\left(t_{n}\right) \leqslant x_{\mathrm{th}}}$ that the state $S$ appears between $t_{0}$ and $t_{\eta-1}$ is $f_{S}=\eta_{S} /\left(\eta_{S}+\eta_{\bar{S}}\right)$ Similarly, the frequency $f_{\bar{S}}=f_{x\left(t_{n}\right)>x_{\mathrm{th}}}$ is $f_{\bar{S}}=\eta_{\bar{S}} /\left(\eta_{S}+\eta_{\bar{S}}\right)$.

\section{B. Communication Errors}

Let $S_{i, 0}[n]=y_{i}[n]$ be the signal sent by sensor $i$ and $S_{i, j}[n]$ be the state at the $j$ th level with $j=1, \ldots, M$ communication hop. At every hop, relay nodes forward their state $S_{i, j}[n]$ to the next one. The probability tree of the state of sensor $i$ is presented in Fig. 2, where the initial state $S_{i, 0}[n]$ is equal to the state $y_{i}[n]$ after the sensing procedure and $S_{i, j}[n]$ is the state at the $j$ th level.

We assume here a binary symmetric channel where the output is different from the input with probability $p_{j}$ for the $j$ th level of the tree. We assume that the error events are independent at each level and time-steps $t_{n}$, which allows for dropping the index $n$. Then, the state $S_{i, j}=S_{i, j-1}$ with probability $1-p_{j}$ and $S_{i, j} \neq S_{i, j-1}$ with probability $p_{j}$. Fig. 3 represents the state transition diagram of this channel.

Definition 2: A communication error event $s_{i}[n] \neq y_{i}[n]$ related to sensor $i$ and $t_{n}$ occurs after $M$ hops if an odd number of errors $S_{i, j} \neq S_{i, j-1}$ had happened for $j=1, \ldots, M$.
Definition 3: Let us define the set of all possible error events for an $M$-hop link as $\mathcal{C}_{M}=$ $\{\{\},\{1\},\{2\}, \ldots,\{1,2\},\{1,3\}, \ldots,\{1,2, \ldots, M\}\}$, which contains $2^{M}$ elements that refer to the index $j=1, \ldots, M$. Let $\mathcal{C}_{M \text {,odd }} \subset \mathcal{C}_{M}$ denote the subset of index with odd cardinality, which is composed by $2^{M-1}$ elements. The subset $\mathcal{C}_{M \text {,odd }}^{k} \subset \mathcal{C}_{M \text {,odd }}$ with $k=1, \ldots, 2^{M-1}$ denotes each one of the $k$ subsets of $\mathcal{C}_{M \text {,odd }}$ such that $\bigcup \mathcal{C}_{M \text {,odd }}^{k}=\mathcal{C}_{M \text {,odd. }}$ The complement $\overline{\mathcal{C}}_{M \text {,odd }}^{k}$ is defined in relation to $\{1,2, \ldots, M\}$ such that $\mathcal{C}_{M \text {,odd }}^{k}+\overline{\mathcal{C}}_{M \text {,odd }}^{k}=\{1,2, \ldots, M\}$.

Let us now exemplify the construction of those sets when $M=3$. Following the procedure presented in Definition 3, $\mathcal{C}_{3}=\{\{\},\{1\},\{2\},\{3\},\{1,2\},\{1,3\},\{2,3\},\{1,2,3\}\}$, which has eight $\left(2^{3}\right)$ elements. The subset is then $\mathcal{C}_{3 \text {,odd }}=$ $\{\{1\},\{2\},\{3\},\{1,2,3\}\}$, having then four $\left(2^{2}\right)$ elements. Using these sets, we have $\mathcal{C}_{3 \text {,odd }}^{1}=\{1\}$ and $\overline{\mathcal{C}}_{3 \text {,odd }}^{1}=\{2,3\}$, $\mathcal{C}_{3, \text { odd }}^{2}=\{2\}$ and $\overline{\mathcal{C}}_{3, \text { odd }}^{2}=\{1,3\}, \mathcal{C}_{3, \text { odd }}^{3}=\{3\}$ and $\overline{\mathcal{C}}_{3, \text { odd }}^{3}=$ $\{1,2\}$, and $\mathcal{C}_{3, \text { odd }}^{4}=\{1,2,3\}$ and $\overline{\mathcal{C}}_{3, \text { odd }}^{4}=\{\}$.

Theorem 1: The communication error probability $P\left(s_{i}[n] \neq y_{i}[n]\right)$ related to sensor $i$ and $t_{n}$ over $M$ hops is given by:

$P\left(s_{i}[n] \neq y_{i}[n]\right)=\sum_{k=1}^{2^{M-1}}\left(\prod_{i \in \mathcal{C}_{M, \text { odd }}^{k}} p_{i}\right)\left(\prod_{j \in \overline{\mathcal{C}}_{M, \text { odd }}^{k}}\left(1-p_{j}\right)\right)$.

Proof: From Lemma 2 we know an error event $s_{i}[n] \neq y_{i}[n]$ occurs whenever an odd number of error events $S_{i, j} \neq S_{i, j-1}$ happens for $j=1, \ldots, M$. To compute the probability of such events, we need to use the law of total probability knowing that the error events at each level and in different measurement instants $t_{n}$ are independent. Using Definition 3 to characterize the subsets containing the odd number of error events, we obtain (2).

Remark 2: If $p_{j}=p$ for all $j=1, \ldots, M$, then

$$
P\left(s_{i}[n] \neq y_{i}[n]\right)=\frac{1-(1-2 p)^{M}}{2},
$$

which is known result for cascade of binary symmetric channels [8].

\section{Decision Function}

Lemma 1: A decision error event $\hat{\theta}[n] \neq \theta[n]$ at fusion center depends on the actual state $\theta[n]$ and the decision function $\hat{\theta}[n]=g\left(s_{1}[n], \ldots, s_{N}[n]\right)$ as follows.

- OR: If $\theta[n]=0$, then at least one signal $s_{i}[n]=1$ with $i \in \mathcal{N}$ leads to $\hat{\theta}[n]=1 \neq \theta[n]$. If $\theta[n]=1$, then an error event $\hat{\theta}[n]=0 \neq \theta[n]$ only occurs when all signals $s_{i}[n]=0$.

- AND: If $\theta[n]=0$, then an error event $\hat{\theta}[n]=1 \neq \theta[n]$ only occurs when all signals $s_{i}[n]=1$. If $\theta[n]=1$, then at least one signal $s_{i}[n]=0$ with $i \in \mathcal{N}$ leads to $\hat{\theta}[n]=0 \neq \theta[n]$.

- $K$-OUT-OF- $N$ : If $\theta[n]=0$, an error event $\hat{\theta}[n]=1 \neq \theta[n]$ occurs when at least $K$ out of $N$ signals $s_{i}[n]=1$. If $\theta[n]=1$, an error event $\hat{\theta}[n]=0 \neq \theta[n]$ 
occurs when at least $K$ out of $N$ signals $s_{i}[n]=0$. If $N$ is an even number and $K=N / 2$, the event where $N / 2$ signals are $s_{i}[n]=0$ and the other $N / 2$ are $s_{i}[n]=1$ may occur and the decision will be randomized such that $\hat{\theta}[n]=0$ or $\hat{\theta}[n]=1$ with $50 \%$ of chance.

- MAJORITY: This rule is a specific case of $K$-OUT-OF- $N$ when $K=\lceil N / 2\rceil$, where $\lceil a\rceil: \mathbb{R} \rightarrow \mathbb{Z}$ represents the ceiling function such that it maps the real number $a$ to its smallest following integer.

Corollary 1: The AND and OR decision functions are conditional versions of $K$-OUT-OF- $N$ rule as follows.

- OR: If $\theta[n]=0$, the error event is equivalent to 1 -OUT-OF- $N$ rule. If $\theta[n]=1$, it is equivalent to $N$-OUT-OF- $N$.

- AND: If $\theta[n]=0$, the error event is equivalent to $N$-OUT-OF- $N$ rule. If $\theta[n]=1$, it is equivalent to 1-OUT-OF- $N$.

\section{Main Results}

In this section we combine the results previously presented to derive the main results of this paper, namely the average error probability given in (1) for the OR, AND, $K$-OUT-OF- $N$ and MAJORITY decision rules as a function of the number of sensors $N$, number of hops $M$ and other system variables.

Lemma 2: The probability $P_{\mathrm{e}, K, S}[n]$ that $K$ out of $N$ sensors experience error events $s_{i}[n] \neq \theta[n]$ at $x\left(t_{n}\right)$ for $x\left(t_{n}\right) \leqslant x_{\text {th }}$ (i.e. $\theta[n]=S$ ) is:

$$
P_{\mathrm{e}, K, S}[n]=\left(\begin{array}{l}
N \\
K
\end{array}\right)\left(P_{S, \bar{S}}[n]\right)^{K}\left(P_{S, S}[n]\right)^{N-K},
$$

where the probabilities $P_{S, \bar{S}}[n]=P\left(x\left(t_{n}\right) \leqslant x_{\mathrm{th}}\right) P\left(s_{i}[n] \neq\right.$ $\left.y_{i}[n]\right)+P\left(x\left(t_{n}\right)>x_{\mathrm{th}}\right) P\left(s_{i}[n]=y_{i}[n]\right)$ and $P_{S, S}[n]=1-$ $P_{S, \bar{S}}[n]$.

Similarly, the probability $P_{\mathrm{e}, K, \bar{S}}[n]$ that $K$ out of $N$ sensors experience error events $s_{i}[n] \neq \theta[n]$ at $x\left(t_{n}\right)$ for $x\left(t_{n}\right)>x_{\text {th }}$ (i.e. $\theta[n]=\bar{S}$ ) is:

$$
P_{\mathrm{e}, K, \bar{S}}[n]=\left(\begin{array}{l}
N \\
K
\end{array}\right)\left(P_{\bar{S}, S}[n]\right)^{K}\left(P_{\bar{S}, \bar{S}}[n]\right)^{N-K}
$$

where the probabilities $P_{\bar{S}, S}[n]=P\left(x\left(t_{n}\right)>x_{\text {th }}\right) P\left(s_{i}[n] \neq\right.$ $\left.y_{i}[n]\right)+P\left(x\left(t_{n}\right) \leqslant x_{\text {th }}\right) P\left(s_{i}[n]=y_{i}[n]\right)$ and $P_{\bar{S}, \bar{S}}[n]=1-$ $P_{\bar{S}, S}[n]$.

In other words, $P_{\mathrm{e}, K, S}[n]$ represents the probability that the signals related to $K$ sensors are in state $\bar{S}$ and $N-K$ sensors are in state $S$ when arriving at the fusion center, given that $\theta[n]=S$ (i.e. $\left.x\left(t_{n}\right) \leqslant x_{\text {th }}\right)$.

Definition 4: Let $\operatorname{Av}(\cdot, \cdot)$ be the average operator such that

$$
\operatorname{Av}(v[n], \mathcal{V})=\frac{1}{\#(v[n])} \sum_{n \in \mathcal{V}} v[n],
$$

where $v[n]$ is a list of numbers, \# $(v[n])$ represents its cardinality and $\mathcal{V}$ is the set containing the indexes related to each one of the \#(v[n]) elements of $v[n]$.

Theorem 2: If the state $S=0$ (i.e. $\theta[n]=0$ if $x\left(t_{n}\right) \leqslant x_{\text {th }}$; refer to Definition 1) the expected decision error probability $P_{\mathrm{e}, \mathrm{OR}, 0}$ introduced in (1) using OR decision rule and $N$ sensors is

$$
P_{\mathrm{e}, \mathrm{OR}, 0}=f_{0} \operatorname{Av}\left(1-P_{\mathrm{e}, N, 0}[n], \mathcal{S}_{0}\right)+f_{1} \operatorname{Av}\left(P_{\mathrm{e}, N, 1}[n], \mathcal{S}_{1}\right),
$$

where $f_{0}$ and $f_{1}$ are given in Definition 1 , and $\mathcal{S}_{0}$ and $\mathcal{S}_{1}$ denote the set containing the indexes related to $S=0$ and $S=1$

Similarly, the probability $P_{\mathrm{e}, \text { AND }, 0}$ using AND decision is:

$P_{\mathrm{e}, \mathrm{AND}, 0}=f_{0} \operatorname{Av}\left(P_{\mathrm{e}, N, 0}[n], \mathcal{S}_{0}\right)+f_{1} \operatorname{Av}\left(1-P_{\mathrm{e}, N, 1}[n], \mathcal{S}_{1}\right)$.

For the $K$-OUT-OF- $N$ rule except when both $N$ is even and $K=N / 2$, the probability $P_{\mathrm{e}, K, 0}$ is:

$$
P_{\mathrm{e}, K, 0}=\sum_{k=K}^{N} f_{0} \operatorname{Av}\left(P_{\mathrm{e}, k, 0}[n], \mathcal{S}_{0}\right)+f_{1} \operatorname{Av}\left(P_{\mathrm{e}, k, 1}[n], \mathcal{S}_{1}\right) .
$$

For MAJORITY and $N$ odd, the probability $P_{\mathrm{e}, \mathrm{MAJ}, 0}^{\mathrm{odd}}$ is:

$$
P_{\mathrm{e}, \mathrm{MAJ}, 0}^{\text {odd }}=\sum_{k=\lceil N / 2\rceil}^{N} f_{0} \mathrm{Av}\left(P_{\mathrm{e}, k, 0}[n], \mathcal{S}_{0}\right)+f_{1} \mathrm{Av}\left(P_{\mathrm{e}, k, 1}[n], \mathcal{S}_{1}\right) \text {. }
$$

For $N$ being even, the error probability $P_{\mathrm{e}, \mathrm{MAJ}, 0}^{\mathrm{even}}$ is:

$$
\begin{aligned}
P_{\mathrm{e}, \mathrm{MAJ}, 0}^{\mathrm{even}}=P_{\mathrm{e}, \mathrm{MAJ}, 0}^{\mathrm{odd}}- & \left(\frac{f_{0}}{2} \operatorname{Av}\left(P_{\mathrm{e}, N / 2,0}[n], \mathcal{S}_{0}\right)\right. \\
& \left.+\frac{f_{1}}{2} \operatorname{Av}\left(P_{\mathrm{e}, N / 2,1}[n], \mathcal{S}_{1}\right)\right) .
\end{aligned}
$$

If $S=1$ such that $\theta[n]=1$ is associated to the $x\left(t_{n}\right) \leqslant x_{\mathrm{th}}$, then $P_{\mathrm{e}, \mathrm{OR}, 1}=P_{\mathrm{e}, \mathrm{AND}, 0}, P_{\mathrm{e}, \mathrm{AND}, 1}=P_{\mathrm{e}, \mathrm{OR}, 0}$, $P_{\mathrm{e}, K, 1}=P_{\mathrm{e}, K, 0}$ and $P_{\mathrm{e}, \mathrm{MAJ}, 1}=P_{\mathrm{e}, \mathrm{MAJ}, 0}$.

Proof: To compute the average error probability $P_{\mathrm{e}}$ given in (1), we first need to compute the probability of error events for each decision rule (described in Lemma 1), knowing the value of $\theta[n]$. We then use the fact that $\theta[n]=0$ if $x\left(t_{n}\right) \leqslant x_{\text {th }}$ (i.e. $S=0$ ), Lemma 2 and Corollary 1 to find probabilities for every measurement. To obtain $P_{\mathrm{e}}$, we compute the average error probabilities for $\theta[n]=0$ or $\theta[n]=1$ using Definition 4 and their respective frequencies $f_{0}$ and $f_{1}$ (Definition 1).

By De Morgan's law, which says that $\bar{A}+\bar{B}=\overline{A \cdot B}$ for any Boolean variables $A$ and $B$, we find that $P_{\mathrm{e}, \mathrm{OR}, 1}=P_{\mathrm{e}, \mathrm{AND}, 0}$ and $P_{\mathrm{e}, \mathrm{AND}, 1}=P_{\mathrm{e}, \mathrm{OR}, 0}$ when considering $S=1$ is related to $x\left(t_{n}\right) \leqslant x_{\mathrm{th}}$. The $K$-OUT-OF- $N$ and MAJORITY rules, in turn, are actually independent of how $S$ is assigned so $P_{\mathrm{e}, K, S=0}=P_{\mathrm{e}, K, S=1}$ and $P_{\mathrm{e}, \mathrm{MAJ}, 1}=P_{\mathrm{e}, \mathrm{MAJ}, 0}$.

Remark 3: These results can be written in terms of type I (false-positive) and type II (false-negative) errors. For $S=0$, the type I error probability is given ${ }^{1}$ :

- OR: $\operatorname{Av}\left(1-P_{\mathrm{e}, N, 0}[n], \mathcal{S}_{0}\right)$,

- AND: $\operatorname{Av}\left(P_{\mathrm{e}, N, 0}[n], \mathcal{S}_{0}\right)$,

- $K$-OUT-OF- $N: \sum_{k=K}^{N} \operatorname{Av}\left(P_{\mathrm{e}, k, 0}[n], \mathcal{S}_{0}\right)$.

\footnotetext{
${ }^{1}$ The results for MAJORITY is a special case of $K$-OUT-OF- $N$.
} 
Similarly, the type II error probability is given by:

- OR: $\operatorname{Av}\left(P_{\mathrm{e}, N, 1}[n], \mathcal{S}_{1}\right)$

- AND: $\operatorname{Av}\left(1-P_{\mathrm{e}, N, 1}[n], \mathcal{S}_{1}\right)$,

- $K$-OUT-OF- $N: \sum_{k=K}^{N} \operatorname{Av}\left(P_{\mathrm{e}, k, 1}[n], \mathcal{S}_{1}\right)$.

Corollary 2: The asymptotic behavior of the error probability $P_{\mathrm{e}}$ with $N$ for $S=0$ and the different rules is:

$$
\begin{aligned}
\lim _{N \rightarrow \infty} P_{\mathrm{e}, \mathrm{OR}, 0} & =f_{0}, \\
\lim _{N \rightarrow \infty} P_{\mathrm{e}, \mathrm{AND}, 0} & =f_{1}, \\
\lim _{N \rightarrow \infty} P_{\mathrm{e}, K}<\lceil N / 2\rceil, 0 & =1, \\
\lim _{N \rightarrow \infty} P_{\mathrm{e}, K \geqslant\lceil N / 2\rceil, 0} & =0, \\
\lim _{N \rightarrow \infty} P_{\mathrm{e}, \mathrm{MAJ}, 0} & =0 .
\end{aligned}
$$

Remark 4: When $N \rightarrow \infty$ and $S=0$, the type I error probability for OR rule tends to 1 , while the type II to 0 . Conversely, when AND rule is considered, the type I error probability for OR rule tends to 0 , while the type II to 1 . Therefore, OR always tends to decide 1, while AND 0.

\section{NUMERICAL ANALYSIS}

To illustrate our framework in a specific setting, let us consider the noisy version of $x(t)$ observed by sensor $i$ such that $x_{i}\left(t_{n}\right)=x\left(t_{n}\right)+n_{i}\left(t_{n}\right)$ where $n_{i}\left(t_{n}\right)$ is related to an additive Gaussian noise with mean $\mu$ and variance $\sigma^{2}$, defining $y_{i}[n]$. Then, the probability $P\left(x_{i}\left(t_{n}\right) \leqslant x_{\text {th }}\right)$ that the event $x_{i}\left(t_{n}\right) \leqslant x_{\text {th }}$ occurs is:

$$
P\left(x_{i}\left(t_{n}\right) \leqslant x_{\text {th }}\right)=\frac{1}{2}\left(1+\operatorname{erf}\left(\frac{x_{\text {th }}-x\left(t_{n}\right)-\mu}{\sigma \sqrt{2}}\right)\right) .
$$

Similarly, the probability $P\left(x_{i}\left(t_{n}\right)>x_{\mathrm{th}}\right)$ is:

$$
P\left(x_{i}\left(t_{n}\right)>x_{\mathrm{th}}\right)=1-\frac{1}{2}\left(1+\operatorname{erf}\left(\frac{x_{\mathrm{th}}-x\left(t_{n}\right)-\mu}{\sigma \sqrt{2}}\right)\right) .
$$

We study in this section the effect of the number of sensors $N$, the number of hops $M$ and the channel error probability for an input signal $x(t)=\sin (12 \pi t / \eta)+\cos (20 \pi t / \eta)+$ $\sin (26 \pi t / \eta)+3$ with $\eta=10^{4}$ and different thresholds $x_{\text {th }}$. We assume that the sensors' observations are affected by additive Gaussian noise with mean $\mu=0$ and variance $\sigma^{2}=1$ and all results have been obtained using $S=0$ such that $\theta[n]=0$ if $x\left(t_{n}\right) \leqslant x_{\text {th }}$. The results are only presented for OR, AND and MAJORITY rules - special variations of the $K$-OUT-OF- $N$, as stated in Theorem 2 and Corollary 1. It is worth mentioning that, although this setting is somehow arbitrary, the analytic framework proposed here can be applied to different scenarios; the computational experiments presented in this section are coded in python language using IPython framework and are available at [28].

Before starting, we would like to explain our choice of the input signal $x(t)$. Our idea was to have a positive deterministic signal, limited in amplitude, that has different peaks and a visually "interesting" behavior. This allows us to see non-linear effects of changes in $x_{\text {th }}$. Our choice $x(t)=\sin (12 \pi t / \eta)+\cos (20 \pi t / \eta)+\sin (26 \pi t / \eta)+3$, whose
TABLE II

ERror Probability for the SNAPShot PRESENTEd IN Fig. 4

\begin{tabular}{|c|c|c|c|}
\hline Decision rule & Analytic & \multicolumn{2}{|c|}{ Simulation } \\
\hline$g$ & $P_{\mathrm{e}}$ & $P_{\mathrm{e}}(\eta=300)$ & $P_{\mathrm{e}}\left(\eta=10^{5}\right)$ \\
\hline \hline OR & 0.383 & 0.366 & 0.381 \\
\hline AND & 0.101 & 0.090 & 0.101 \\
\hline MAJORITY & 0.129 & 0.136 & 0.125 \\
\hline
\end{tabular}

amplitude varies from 0 to 6 is illustrated in the first plot of Fig. 4, but for $\eta=300$ and assuming $M=1$ and $p_{1}=0.1$, $N=3$ with additive Gaussian noise $\left(\mu=0, \sigma^{2}=1\right)$.

The top plot represents the signal $x(t)$ while the actual system state $\theta[n]$ associated with the event $x\left(t_{n}\right)>x_{\text {th }}$ is shown next. We present in the third plot the estimations $x_{i}\left(t_{n}\right)$ from the three sensors based on their noisy version of $x(t)$, followed by their respective states $y_{i}[n]$. The three received signals $s_{i}[n]$ at the fusion center, after passing through the communication link, are presented next. The last plots represent the decisions using OR, AND and MAJORITY.

Table II compares the simulated and analytic error probabilities for this example, which for better visualization only considers $\eta=300$. Notice that for this particular example AND rule has better performance compared with the other two. As we shall see later, AND rule leads to small error probabilities when the number of sensors is low.

Fig. 5 illustrates how the average error probability $P_{\mathrm{e}}$ using OR, AND and MAJORITY rules varies with the number $N$ of sensors making measurements when the threshold that defines the event $\mathcal{E}$ is $x_{\text {th }}=4.5$. The plot shows that: $(i)$ OR rule has a high error probability, which increases with $N$, (ii) AND rule has the best performance for small values of $N$, and (iii) MAJORITY is the best choice when $N$ grows such that $P_{\mathrm{e}}$ tends to 0 . To analyze these facts, we need to deal with $\theta[n]$. From Theorem 2 and Corollary 2, the frequency of $\theta[n]=0$ and $\theta[n]=1$ determines the performance of OR and AND so the former outperforms when $\theta[n]=0$ is more frequent, and vice-versa. In our example, the frequencies are: $f_{0}=0.8747$ and $f_{1}=0.1253$, providing their asymptotic limit.

For MAJORITY, the asymptotic performance is independent of such frequencies. When a small number of sensors is considered, however, it does not provide the best performance since an error in more than $N / 2$ signal is not rare. In this case, AND is the best, even working below its asymptotic limit. This happens due to the way that the logic operation AND works, balancing the error events when $\theta[n]=0$ and $\theta[n]=1$.

To get more insights on the system performance, Figs. 6, 7 and 8 show the average error probability as a function $N$ for $x_{\text {th }}=5.5\left(f_{0}=0.979\right.$ and $\left.f_{1}=0.021\right)$, $x_{\text {th }}=3\left(f_{0}=0.4992\right.$ and $\left.f_{1}=0.5008\right)$ and $x_{\text {th }}=1.5\left(f_{0}=\right.$ 0.1128 and $f_{1}=0.8872$ ), respectively. In the scenario where $x_{\text {th }}=5.5$, a similar behavior to the $x_{\text {th }}=4.5$ is observed, but with the AND rule having a better performance due to the smaller frequency $f_{1}$ of events $\theta[n]=1$. On the other hand, when $x_{\text {th }}=1.5$, the performance of the OR and AND rules switches in relation to when $x_{\mathrm{th}}=4.5$ as far as the frequencies $f_{0}$ and $f_{1}$ have also switched; now OR works better because $\theta[n]=0$ is much more frequent. When the 

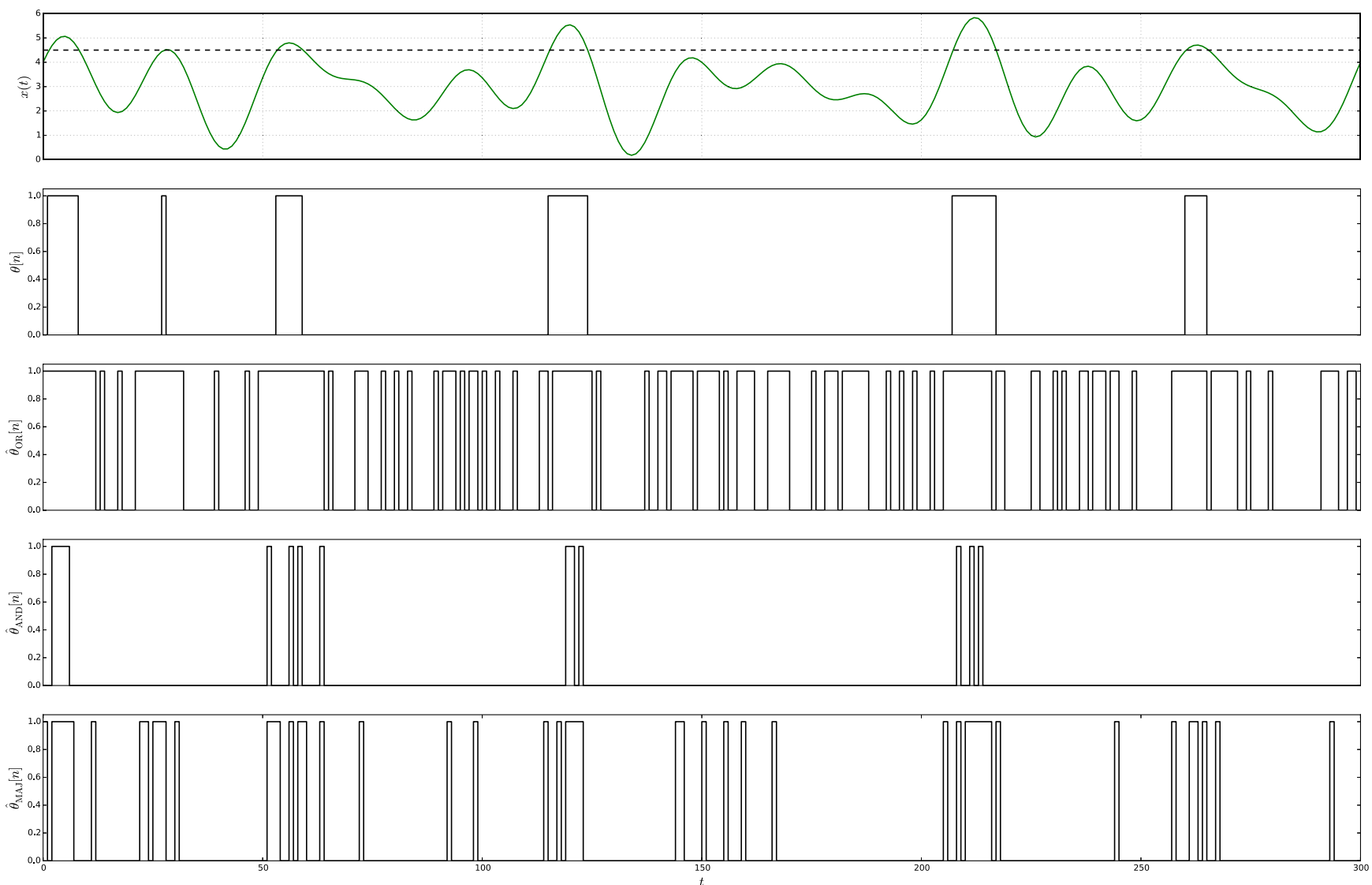

Fig. 4. Numerical example of the proposed scenario for the signal $x(t)=\sin (12 \pi t / \eta)+\cos (20 \pi t / \eta)+\sin (26 \pi t / \eta)+3$, which has a mean value of 3 and $\eta=300, x_{\text {th }}=4.5$ (represented by the dashed line in the first and third plots, and leading to $\eta_{0}=262$ and $\eta_{1}=38$ ), $N=3$ (three sensors) and $M=1$ (one hop). Sensor $i$ sets its state $y_{i}[n]$ at $t_{n}$ based on the noisy version of signal denoted by $x_{i}\left(t_{n}\right)=x\left(t_{n}\right)+n_{i}\left(t_{n}\right)$ where $n_{i}\left(t_{n}\right)$ is related to an additive Gaussian noise with mean $\mu=0$ and variance $\sigma^{2}=1$. The error probabilities associated with the sensing procedure are given in 17 and 18 . After the sensing procedure, signal $y_{i}[n]$ is sent via a binary symmetric channel with error probability $p_{1}=0.1$, yielding a new signal $s_{i}[n]$. At the fusion center, a decision is done based on $s_{i}[n]$ and the logic operations OR, AND and MAJORITY. Table II presents the error probabilities associated with this scenario.

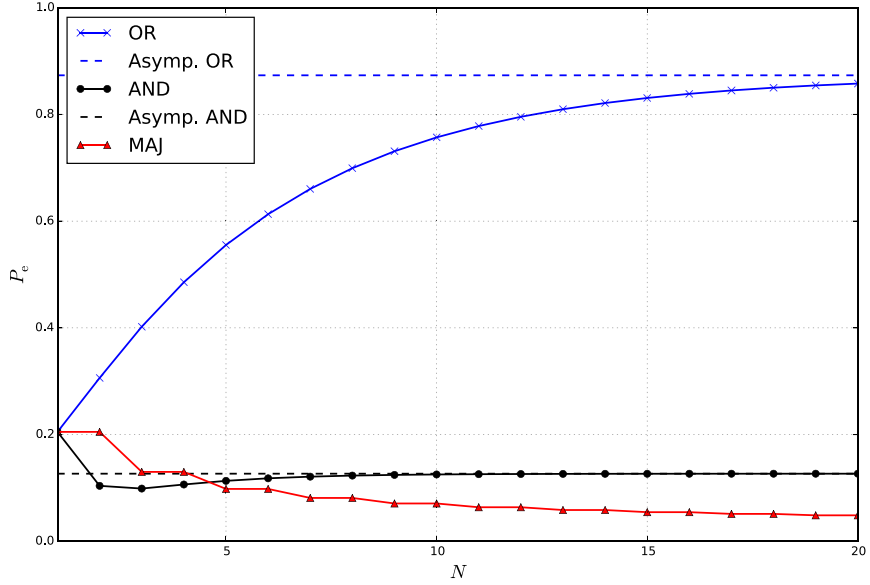

Fig. 5. Average error probability $P_{\mathrm{e}}$ given in Theorem 2 as a function of the number of sensors $N$ for OR, AND and MAJORITY decision rules, threshold $x_{\text {th }}=4.5, M=1$ and $p_{1}=0.1$. The total number of samples is $\eta=10^{4}$ where $\eta_{0}=8747$ and $\eta_{1}=1253$.

$\theta[n]$ is more evenly distributed, illustrated in the scenario where $x_{\text {th }}=3$, AND and OR are equivalent and their error probability tends to 0.5 (which is basically a random guess

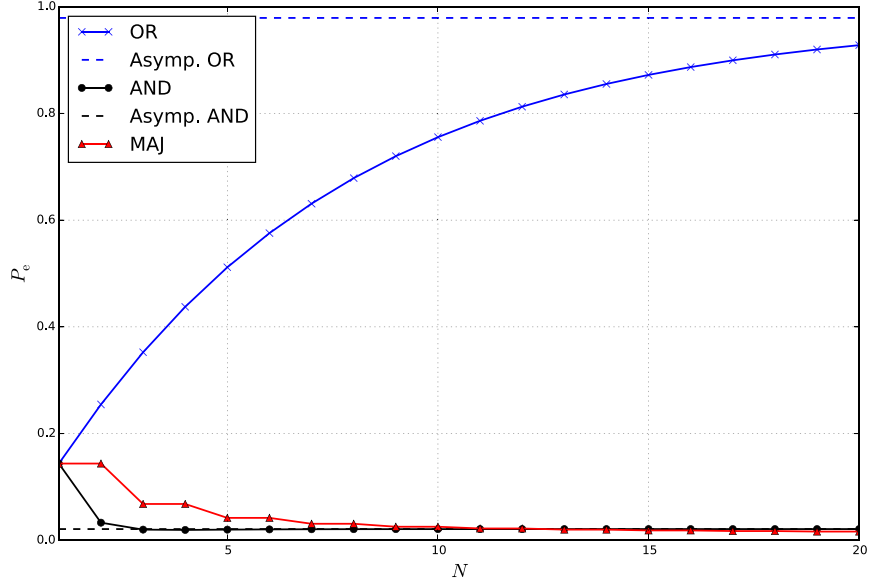

Fig. 6. Average error probability $P_{\mathrm{e}}$ given in Theorem 2 as a function of the number of sensors $N$ for OR, AND and MAJORITY decision rules, threshold $x_{\text {th }}=5.5, M=1$ and $p_{1}=0.1$. The total number of samples is $\eta=10^{4}$ where $\eta_{0}=9790$ and $\eta_{1}=210$.

of the input state). In all scenarios, the MAJORITY rule maintains its asymptotic optimal performance, working better and better when the number of sensors grows. 


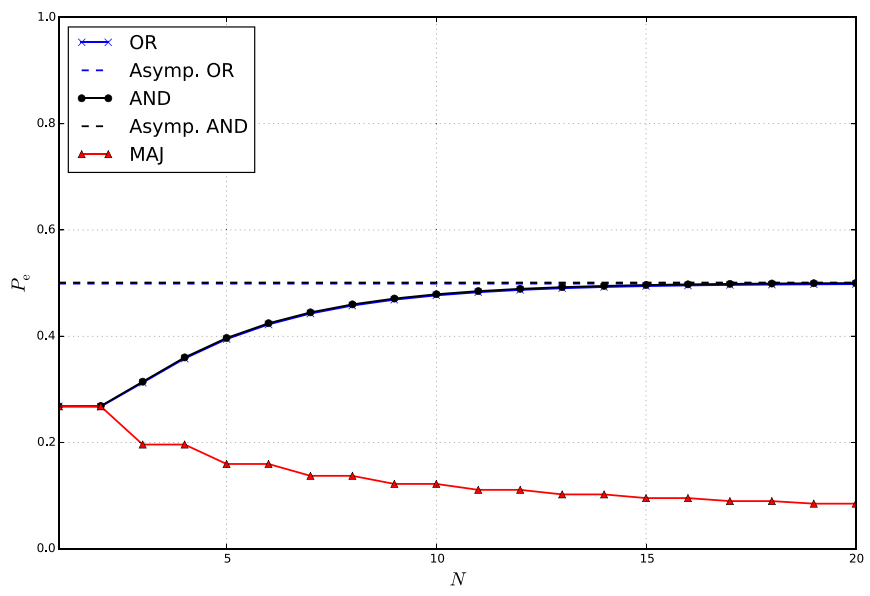

Fig. 7. Average error probability $P_{\mathrm{e}}$ given in Theorem 2 as a function of the number of sensors $N$ for OR, AND and MAJORITY decision rules, threshold $x_{\text {th }}=3, M=1$ and $p_{1}=0.1$. The total number of samples is $\eta=10^{4}$ where $\eta_{0}=4992$ and $\eta_{1}=5008$.

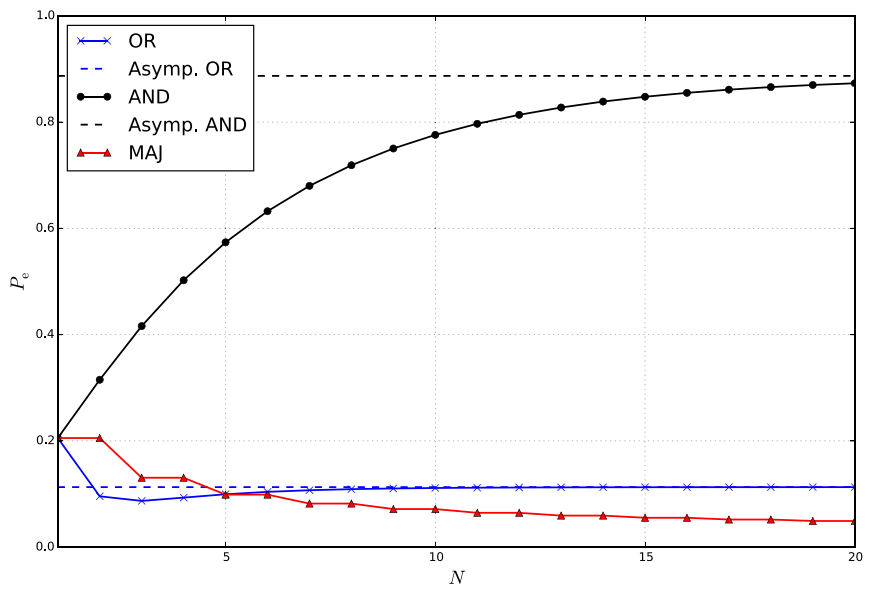

Fig. 8. Average error probability $P_{\mathrm{e}}$ given in Theorem 2 as a function of the number of sensors $N$ for OR, AND and MAJORITY decision rules, threshold $x_{\text {th }}=1.5, M=1$ and $p_{1}=0.1$. The total number of samples is $\eta=10^{4}$ where $\eta_{0}=1128$ and $\eta_{1}=8872$.

Fig. 9 presents the effects of the number of hops on the average error probability for $x_{\mathrm{th}}=4.5$ and $N=3$, considering that every one of the $M$ hops is modeled as a binary symmetric channel with the same error probability $p=0.1$ so that the equivalent channel has the error probability given by Corollary 2. As one would expect, the increase of the number of hops $M$ also increases the average error probability, regardless of the decision rule. For the setting considered here, the AND rule seems more robust against the increase of $M$, so that the error probability associated to it grows slower than the other two options. Once again, this fact occurs due to the way AND balances the error probabilities for the two possible values of $\theta[n]$, given more weight (in proportion to its occurrence) to less frequent error events. The OR rule, on the other hand, has an overall poor performance because it balances the error probability in the opposite way, which leads to even worse error probabilities. The MAJORITY rule appears to be more susceptible to the increase of $M$ than AND, which indicates that the increase of the equivalent channel

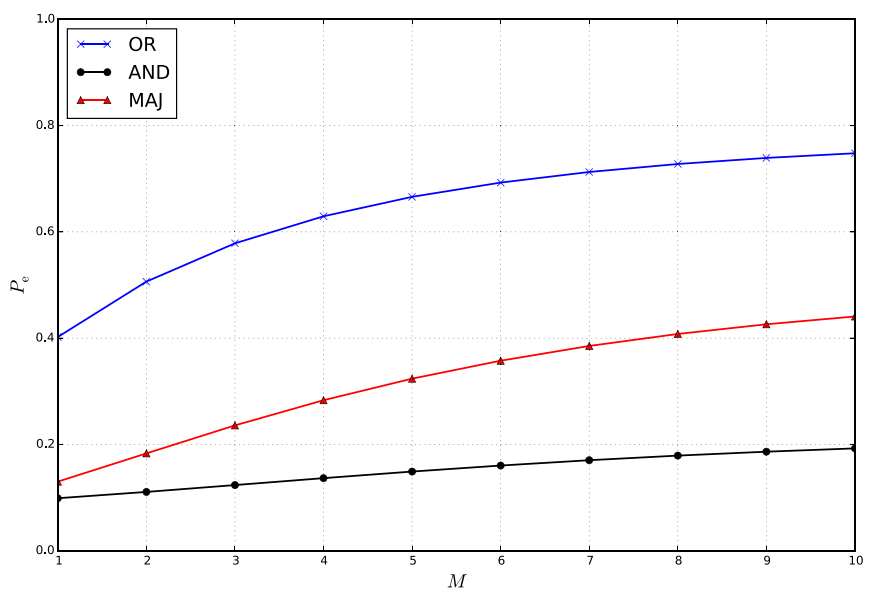

Fig. 9. Average error probability $P_{\mathrm{e}}$ given in Theorem 2 as a function of the number of hops $M$ considering $p_{j}=p=0.1$ for OR, AND and MAJORITY rules, threshold $x_{\text {th }}=4.5$ and $N=3$. The equivalent channel probability after $M$ hops is given in Corollary 2.

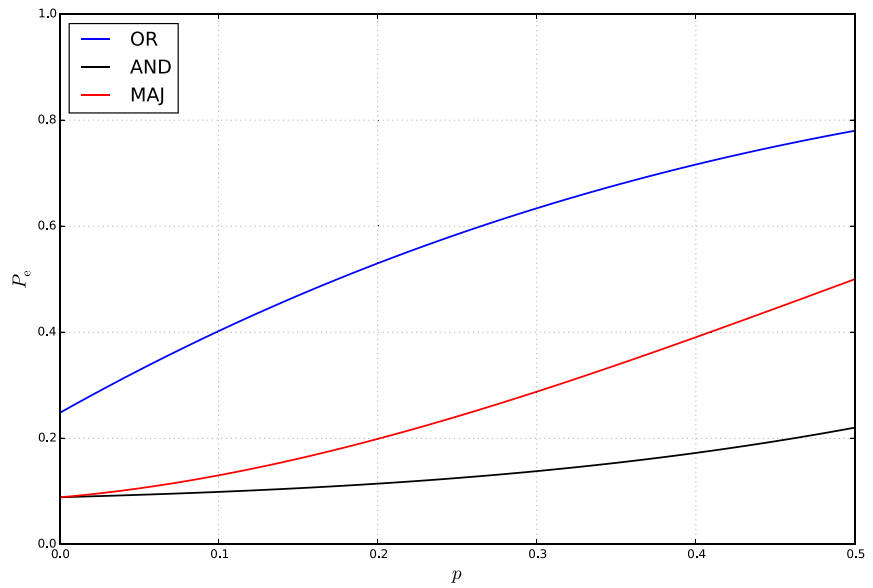

Fig. 10. Average error probability $P_{\mathrm{e}}$ given in Theorem 2 as a function of channel error probability $p_{1}$ considering only 1 -hop transmission (i.e. $M=1$ ) for OR, AND and MAJORITY rules, threshold $x_{\text {th }}=4.5$ and $N=3$.

error probability when $M$ grows seems to affect more the former.

To better understand this fact, we present in Fig. 10 how the average error probability varies with the channel error probability $p_{1}$ when $x_{\mathrm{th}}=4.5, M=1$ and $N=3$. We now can see clearer that the MAJORITY rule is indeed more susceptible to worse channel conditions than the AND rule for the scenario under analysis. As presented in Theorem 2, MAJORITY, different from the latter rule, does not favor the error events in different ways so that when the channel error probability increases, it will increase in the same proportion for both the more and less frequent states. Consequently, although MAJORITY asymptoticly outperforms the others in terms of the number of sensors, it is much more vulnerable to an increase of the channel error probabilities.

\section{FinAl REMARKS}

In this paper, we analyzed different ways that one could design a relatively simple WSN based on three phases, namely sensing, communication and decision. Different from the 
literature of distributed sensing and estimation, we targeted at implementing simple decision rules, regardless of their optimality. Our idea here was to show that it is possible to attain low error probabilities using a simple threshold based quantizer, a limited bandwidth of 1 bit and low complexity decision rules such as AND, OR and MAJORITY.

If the occurrence of the event is rare and associated with the state " 1 ", the AND rule can lead to a low error probability with a small number of sensors, although MAJORITY can asymptotically reach 0 -error probability for a large the number of sensors. If its occurrence is more equally distributed, then AND and OR rules have a poor performance while the MAJORITY is better than the other options but still requiring a relatively large number of sensors. We also show that the MAJORITY rule is more susceptible to channel errors than the AND rule, reflecting the way that it balances the error events with the input state frequencies.

In any case, our results indicate a simple and cheap way to implement a WSN when the application does not have strict requirements. Our plan is to extend these results by considering erasure channels as in [14] and advanced relay strategies as in [29] and [30], assuming the nodes follow a specific spatial distribution as in [19]. Sensors experiencing different input signals (e.g. smart-metering or sensors in different rooms) also constitute an interesting extension of the present work. Other promising direction is to individually analyze the types I and II error probabilities such keeping one of them as a fixed target (e.g. applications that require a very low false negative probability, while false positives are unconstrained).

\section{REFERENCES}

[1] J. Manyika, et al., "Unlocking the potential of the Internet of Things," McKinsey Global Institute, New York, NY, USA, Tech. Rep., 2015.

[2] C. Perera, C. H. Liu, S. Jayawardena, and M. Chen, "A survey on Internet of Things from industrial market perspective," IEEE Access, vol. 2, pp. 1660-1679, Jan. 2014.

[3] P. Gaj, A. Malinowski, T. Sauter, and A. Valenzano, "Guest editorial: Distributed data processing in industrial applications," IEEE Trans. Ind. Informat., vol. 11, no. 3, pp. 737-740, Jun. 2015.

[4] L. Da Xu, W. He, and S. Li, "Internet of Things in industries: A survey," IEEE Trans. Ind. Informat., vol. 10, no. 4, pp. 2233-2243, Nov. 2014.

[5] S. D. T. Kelly, N. K. Suryadevara, and S. C. Mukhopadhyay, "Towards the implementation of IoT for environmental condition monitoring in homes," IEEE Sensors J., vol. 13, no. 10, pp. 3846-3853, Oct. 2013.

[6] J. N. Tsitsiklis, "Decentralized detection," in Advances in Statistical Signal Processing. Greenwich, CT, USA: JAI Press, 1993.

[7] J.-F. Chamberland and V. V. Veeravalli, "Wireless sensors in distributed detection applications," IEEE Signal Process. Mag., vol. 24, no. 3, pp. 16-25, May 2007.

[8] T. M. Cover and J. A. Thomas, Elements of Information Theory. New York, NY, USA: Wiley, 2012.

[9] E. Ayanoglu, "On optimal quantization of noisy sources," IEEE Trans. Inf. Theory, vol. 36, no. 6, pp. 1450-1452, Nov. 1990.

[10] J. A. Gubner, "Distributed estimation and quantization," IEEE Trans. Inf. Theory, vol. 39, no. 4, pp. 1456-1459, Jul. 1993.

[11] R. Viswanathan and P. K. Varshney, "Distributed detection with multiple sensors-Part I: Fundamentals," Proc. IEEE, vol. 85, no. 1, pp. 54-63, Jan. 1997.

[12] R. S. Blum, S. A. Kassam, and H. V. Poor, "Distributed detection with multiple sensors-Part II: Advanced topics," Proc. IEEE, vol. 85, no. 1, pp. 64-79, Jan. 1997.

[13] A. Sinha, H. Chen, D. G. Danu, T. Kirubarajan, and M. Farooq "Estimation and decision fusion: A survey," Neurocomputing, vol. 71, nos. 13-15, pp. 2650-2656, 2008.
[14] Z. Zhang, E. K. P. Chong, A. Pezeshki, W. Moran, and S. D. Howard, "Detection performance in balanced binary relay trees with node and link failures," IEEE Trans. Signal Process., vol. 61, no. 9, pp. 2165-2177, May 2013.

[15] Z. Zhang, A. Pezeshki, W. Moran, S. D. Howard, and E. K. P. Chong, "Error probability bounds for balanced binary relay trees," IEEE Trans. Inf. Theory, vol. 58, no. 6, pp. 3548-3563, Jun. 2012.

[16] G. Ferrari and R. Pagliari, "Decentralized binary detection with noisy communication links," IEEE Trans. Aerosp. Electron. Syst., vol. 42, no. 4, pp. 1554-1563, Oct. 2006.

[17] J. Ho, W. P. Tay, T. Q. S. Quek, and E. K. P. Chong, "Robust decentralized detection and social learning in tandem networks," IEEE Trans. Signal Process., vol. 63, no. 19, pp. 5019-5032, Oct. 2015.

[18] V. V. Veeravalli, T. Basar, and H. V. Poor, "Minimax robust decentralized detection," IEEE Trans. Inf. Theory, vol. 40, no. 1, pp. 35-40, Jan. 1994.

[19] P. Zhang, I. Nevat, G. W. Peters, G. Xiao, and H.-P. Tan, "Event detection in wireless sensor networks in random spatial sensors deployments," IEEE Trans. Signal Process., vol. 63, no. 22, pp. 6122-6135, Nov. 2015.

[20] P. H. J. Nardelli, C. H. M. de Lima, H. Alves, P. Cardieri, and M. Latva-Aho, "Throughput analysis of cognitive wireless networks with Poisson distributed nodes based on location information," Ad Hoc Netw., vol. 33, pp. 1-15, Oct. 2015. [Online]. Available: http://www.sciencedirect.com/science/article/pii/S1570870515000748.

[21] G. Ferrari, M. Martalo, and R. Pagliari, "Decentralized detection in clustered sensor networks," IEEE Trans. Aerosp. Electron. Syst., vol. 47, no. 2, pp. 959-973, Apr. 2011.

[22] K. Umebayashi, J. J. Lehtomaki, T. Yazawa, and Y. Suzuki, "Efficient decision fusion for cooperative spectrum sensing based on OR-rule," IEEE Trans. Wireless Commun., vol. 11, no. 7, pp. 2585-2595, Jul. 2012.

[23] D. A. Castanon and D. Teneketzis, "Distributed estimation algorithms for nonlinear systems," IEEE Trans. Autom. Control, vol. 30, no. 5, pp. 418-425, May 1985.

[24] A. Ribeiro and G. B. Giannakis, "Bandwidth-constrained distributed estimation for wireless sensor networks-Part I: Gaussian case," IEEE Trans. Signal Process., vol. 54, no. 3, pp. 1131-1143, Mar. 2006.

[25] A. Ribeiro and G. B. Giannakis, "Bandwidth-constrained distributed estimation for wireless sensor networks-Part II: Unknown probability density function," IEEE Trans. Signal Process., vol. 54, no. 7, pp. 2784-2796, Mar. 2006.

[26] A. Dogandžić and B. Zhang, "Distributed estimation and detection for sensor networks using hidden Markov random field models," IEEE Trans. Signal Process., vol. 54, no. 8, pp. 3200-3215, Aug. 2006.

[27] H. Leung, C. Seneviratne, and M. Xu, "A novel statistical model for distributed estimation in wireless sensor networks," IEEE Trans. Signal Process., vol. 63, no. 12, pp. 3154-3164, Jun. 2015.

[28] P. H. J. Nardelli. [Online]. Available: http://nbviewer.ipython.org/gist/ pedrohjn/3139775c440db1d1064f.

[29] H. Alves, R. D. Souza, and G. Fraidenraich, "Outage, throughput and energy efficiency analysis of some half and full duplex cooperative relaying schemes," Trans. Emerg. Telecommun. Technol., vol. 25, no. 11, pp. 1114-1125, Nov. 2014.

[30] C. H. M. de Lima, H. Alves, P. H. J. Nardelli, and M. Latva-Aho. (2016). "Effects of relay selection strategies on the spectral efficiency of wireless systems with half- and full-duplex nodes." [Online]. Available: http://arxiv.org/abs/1507.03761.

Pedro Henrique Juliano Nardelli received the B.S. and M.Sc. degrees in electrical engineering from the State University of Campinas, Brazil, in 2006 and 2008, respectively, and the Ph.D. degree from the University of Oulu, Finland, and State University of Campinas, following a dual-degree agreement, in 2013. He currently holds a post-doctoral position with the University of Oulu, and his studies are mainly focused on the efficiency of wireless networks and spatiotemporal dynamics of complex systems.

Iran Ramezanipour received the B.Sc. degree in electrical and electronic engineering from Karaj Azad University, Iran, in 2011, and the M.Sc. degree in wireless communications engineering from the University of Oulu, Finland, in 2016. She is currently pursuing the Ph.D. degree with the Center for Wireless Communications, University of Oulu, Finland. Her main research areas include communication systems for smart grids and spectrum sharing in smart grids communication systems. 
Hirley Alves received the B.Sc., M.Sc., and D.Sc. degrees from the Federal University of Technology-Paraná, Brazil, in 2010, 2011, and 2015, respectively. He has jointly graduated from the University of Oulu, Oulu, and received the D.Sc. degree. He is currently a Post-Doctoral Researcher with the Centre for Wireless Communications, University of Oulu. His current research focuses on: performance analysis of full-duplex networks and relaying and its applications on $5 \mathrm{G}$, and smart grids.

Carlos H. M. de Lima received the B.Sc. and M.Sc. degrees in electrical engineering from the Federal University of Ceara, Fortaleza, Brazil, in 2002 and 2004, respectively, and the D.Sc. degree in telecommunications engineering from the University of Oulu, Finland, in 2013. From 2000 to 2005 , he was a Research Scientist with the Wireless Telecommunications Research Group, Fortaleza. In 2005, he was a Visiting Researcher with the Ericsson Research Center, Lulea, Sweden, engaged in power control techniques for enhanced high-speed packet access systems. In 2006, he was with the Nokia Institute of Technology, Brazil, engaged in the evaluation of the system performance of WiMAX systems. He is currently an Assistant Professor at Sao Paulo State University, São João da Boa Vista de São Paulo, Brazil, and a member of the Research Staff with the Centre for Wireless Communications, University of Oulu. His research interests include statistical signal processing and analysis of interference networks using stochastic geometry.
Matti Latva-Aho was born in Kuivaniemi, Finland, in 1968. He received the M.Sc., Lic.Tech., and D.Tech. (Hons.) degrees from the University of Oulu, Finland, in 1992, 1996, and 1998, respectively, all in electrical engineering. From 1992 to 1993, he was a Research Engineer with Nokia Mobile Phones, Oulu, Finland. From 1994 to 1998, he was a Research Scientist with the Telecommunication Laboratory, University of Oulu, and the Centre for Wireless Communications. He was the Director of the Centre for Wireless Communications, University of Oulu from 1998 to 2006. He is currently the Department Chair Professor of Digital Transmission Techniques and the Head of the Department for Communications Engineering, University of Oulu. He has authored over 200 conference or journal papers in the field of wireless communications. His research interests are related to mobile broadband wireless communication systems. He has been the TPC Chairman of PIMRC06, the TPC Co-Chairman of ChinaCom07, and the General Chairman of WPMC08. He acted as the Chairman and Vice Chairman of the IEEE Communications Finland Chapter from 2000 to 2003. 